\title{
Primary breast lymphoma - a review of the literature and report of three cases
}

Monika Joks¹, Krzysztof Myśliwiec², Krzysztof Lewandowski

1Lord's Transfiguration Hospital, Poznan, Poland

2Haematology Outpatients Department, State Hospital, Zielona Gora, Poland

${ }^{3}$ Department of Haematology, University of Medical Sciences, Poznan, Poland

Submitted: 23 April 2010

Accepted: 4 August 2010

Arch Med Sci 2011; 7, 1: 27-33

DOI: 10.5114/aoms.2011.20600

Copyright ๑ 2011 Termedia \& Banach

\begin{abstract}
Primary breast lymphoma $(\mathrm{PBL})$ is a rare disease accounting for $0.4-0.5 \%$ of all breast malignancies. Diffuse large B-cell lymphoma ( $\mathrm{DLBCL}$ ) is the most common histological diagnosis. The clinical presentation of PBLs is usually no different from that of carcinoma. In this paper we review the literature on the clinical presentation, diagnosis, prognostic factors and treatment options of PBL. In the light of the information gained we discuss three patients with primary breast lymphoma (one with a central nervous system relapse) who were treated in our department in the years 2002-2007. In conclusion: there is no consensus on the question of how to best treat PBL: chemotherapy, radiotherapy or combined therapy. However, the last approach to be the most successful one. Due to high incidence of central nervous system (CNS) involvement in PBL patients, many authors strongly believe that patients with aggressive forms of PBL should receive CNS infiltration prophylaxis, even in the early stages, as this may improve the outcome and significantly reduce the risk of a CNS disease relapse.
\end{abstract}

Key words: primary breast lymphoma, diffuse large B cell lymphoma, extranodal non-Hodgkin lymphoma.

\section{Introduction}

The term "primary breast lymphoma" (PBL) is used to define a malignant lymphoma primarily occurring in the breast in the absence of previously detected lymphoma localizations [1]. PBL is a rare disease, accounting for only $0.4-0.5 \%$ of all breast malignancies, $0.38-0.7 \%$ of all non-Hodgkin lymphomas (NHL), and $1.7-2.2 \%$ of extranodal NHL. The median age of patients with diagnosed PBL ranges from 60 to 65 years [1-12]. The disease occurs almost exclusively in women. Bilateral breast involvement accounts for $11 \%$ of all breast lymphomas [13] or $5 \%$ according to Ryan et al. [11]. This rare situation is especially observed during pregnancy or postpartum, suggesting that tumour growth is influenced by hormonal stimulation.

Breast lymphoid cells probably originate in mucosa-associated lymphoid tissue (MALT) [14]. PBL may also originate from lymphatic tissue present within the breast adjacent to ducts and lobules, or from intramammary lymph nodes $[15,16]$.

More than $80 \%$ of PBL are B-cell lymphomas, mostly CD20+. The most frequent histopathologic types are: diffuse large B-cell lymphoma (DLBCL)

\author{
Corresponding author: \\ Monika Joks MD \\ Lord's Transfiguration \\ Hospital \\ Szamarzewskiego 84 \\ 69-569 Poznan, Poland \\ Phone: +48 618549350 \\ E-mail: mjoks@tlen.pl
}


which accounts for up to $50 \%$ of all PBL, follicular lymphoma (FL) - 15\%, MALT lymphoma $-12.2 \%$, Burkitt's lymphoma (BL) and Burkitt-like lymphoma $-10.3 \%$ [17]. Other histological types of PBL include marginal zone lymphoma (MZL), small lymphocytic lymphoma (SLL), and anaplastic large cell lymphoma (ALCL).

Diffuse large $B$-cell lymphoma ( $D L B C L)$ is the most common histological diagnosis. These lymphomas have been shown to be of a non-germinal centre B-cell phenotype with a high proliferation index and are thought to be associated with a poor outcome [18]. There is a close association between ALCL and silicone breast implants [19, 20].

Burkitt's lymphoma is observed particularly in pregnant or lactating women and HIV-seropositive patients. The clinical presentation of PBL and the radiological features are usually no different from those of carcinoma of the breast. PBL is usually classified according to the Ann Arbor staging system. Other diagnostic criteria for PBL were described by Wiseman and Liao in 1972 [21]. According to the last classification, the clinical site of presentation is the breast. A history of previous lymphoma or evidence of widespread disease are absent at diagnosis. There is present close association of lymphoma to breast tissue in the pathologic specimen. Ipsilateral lymph nodes may be involved. This definition of PBL comprises only tumours being in stage I (lymphoma limited to the breast) and stage II (lymphoma limited to the breast and axillary lymph nodes) and not to tumours originating from non-breast sites. Here we present 3 cases of patients with PBL ( 2 with DLBCL and 1 with FL) treated in the years 2002-2007.

\section{Epidemiology and clinical manifestation}

A painless mass is the most common presenting sign in PBL occurring in approximately $61 \%$ of cases, especially (48\%) in the external superior quadrant. Other symptoms and signs are the following: local pain $-12 \%$, local inflammation - $11 \%$, palpable lymph nodes $-25 \%$, incidental mammography finding - 12\% [1]. Mammographic findings are nonspecific. Most of the lesions are oval-shaped (71\%) and high-density (90.9\%) masses. The masses revealed by ultrasound examination are single (75\%), circumscribed (50\%), microlobulated (37.5\%) and oval (50\%). The echo pattern of the mass is usually hypoechoic (87\%). No masses have spiculated margins or calcifications [22]. Fine needle aspiration, core biopsy and excisional biopsy are effective techniques used in the evaluation of breast nodules and axillary lymph nodes. However, histological, immunohistochemical and, sometimes, genetic studies are necessary for establishing the diagnosis.

\section{Prognostic factors}

There are many controversies about prognostic factors for patients with PBL. One of these is the histological subtype of PBL. According to Ryan et al. [11], a favourable International Prognostic Index (IPI) score, the use of anthracycline-containing chemotherapy, and radiotherapy (RT) are significantly associated with longer overall survival (OS). Similarly, Jeanneret-Sozzi [1] confirmed, by means of univariate analysis, that favourable prognostic factors are: early stage (IE), conservative surgery, RT administration and combined modality treatment. Multivariate analyses also confirm that the early stage and use of RT are favourable prognostic factors. According to Lin et al. [23], the Ann Arbor stage, the IPI excluding stage, lactic dehydrogenase (LDH) and radiotherapy are significant factors for relapse-free survival. Ganjoo et al. [13], in their analysis of 37 patients with PBL - DLBCL, found that elevated LDH and an Eastern Cooperative Oncology Group (ECOG) performance status of more than 2 adversely correlated with OS. There are also studies where the authors suggest age to be an independent predictor of outcome [8, 24]. Jennings et al. [17] in a Cox regression model, analyzing the tumour size and node status, revealed that the node status is the best single predictor of survival. Contrary to many studies, Aviles et al. [3], in univariate analysis, did not show that any of the following prognostic factors influenced response: event-free survival (EFS), OS: age, tumour size, IPI score or Ann Arbor stage.

\section{Therapeutic options and clinical outcome - review of articles}

Ganjoo et al. [13] searched the Stanford lymphoma database for patients with documented lymphoma involving the breast between 1981 and 2005 and included 37 patients in their analysis. Diagnostic tissue biopsies were obtained, either from the breast mass or from an involved lymph node. DLBCL was the most common histological subtype, being diagnosed in $49 \%$ of their patients; in turn FL and MZL was diagnosed in $38 \%$. Most patients presented with a painless, unilateral breast mass in stage I(E) or II(E), while only one patient had CNS disease. Their DLBCL patients received doxorubicin-based chemotherapy, with 70\% receiving involved-field radiotherapy (IFRT) with doses of 3600-5040 cGy. A single patient additionally received cytotoxic intrathecal therapy. No recurrences occurred in the involved breast. A single parenchymal CNS recurrence was recorded. Among the DLBCL patients, 5-year progression-free survival (PFS) was 61\%, with a median follow-up of 3.8 years (range: 5 months to 19 years) and a 5 -year OS was estimated at $82 \%$. Patients with indolent 
lymphoma had an estimated 5-year PFS of $76 \%$ and an OS of $92 \%$. The authors conclude that DLBCL of the breast can be successfully treated with doxorubicin-based chemotherapy alone or with IFRT.

In 2008, Ryan et al. [11] published the results of a retrospective international study of 204 eligible patients with PBL DLBCL histological subtype attending the International Extranodal Lymphoma Study Group-affiliated institutions from 1980 to 2003. The median age was 64 years, median OS was 8.0 years, and median progression-free survival (PFS) was 5.5 years. There was no benefit from mastectomy, as opposed to biopsy or lumpectomy. At a median follow-up time of 5.5 years, in 35\% of patients the tumour had progressed: $16 \%$ locally in the same or contralateral breast, $5 \%$ in the CNS and $14 \%$ in other extranodal sites. According to the authors, the combination of limited surgery, anthracycline-containing chemotherapy, and involved-field RT produced the best outcome in the pre-rituximab era. In conclusion, they postulated that a combined therapy was the best method of treating patients with PBL. They suggested using prophylaxis to avoid central nervous system involvement.

A similar opinion was expressed by Aviles et al. [3] on the basis of their prospective study. 96 patients in the early stage (I or II according to the Ann Arbor criteria) of PBL, most of them within an intermediate IPI score risk, were included. They were enrolled to receive IFRT with a total dose of 45 Gy, chemotherapy (6 × CHOP-21), or combined therapy ( 6 cycles of CHOP and radiotherapy with a total dose of $30 \mathrm{~Gy}$ ). Surgery was not considered as initial therapy. Those patients who failed to respond to radiotherapy received a chemotherapy regimen. In patients who failed to respond to chemotherapy, radiotherapy was employed at a dose of $45 \mathrm{~Gy}$. If the failure was outside the breast, they were treated with salvage regimens.

Complete remission was confirmed in 20 of the 30 patients treated with radiotherapy, in 19 of the 32 who were treated with chemotherapy and in 30 of the 34 in the combined arm $(p<0.01)$. At a medium follow-up of 10 years' event-free survival (EFS) was $50 \%, 57 \%$ and $83 \%$, respectively $(p<0.01)$. Overall survival at 10 years was $50 \%$, $50 \%$ and $76 \%(p<0.01)$, respectively. Treatmentrelated acute toxicity was mild, and no second neoplasm or acute leukaemia was observed.

The most common site of relapse of PBL was the CNS. Such relapses were treated with high-dose methotrexate (HD MTX) $6 \mathrm{~g} / \mathrm{m}^{2}$ administered every 28 days for at least four cycles. Patients who had a relapse outside the CNS were treated with intensive chemotherapy, including ASCT. A second response was observed in 6 of 11 patients with relapse in the CNS, and 8 of 12 patients with another site of relapse. All the patients with relapse in the CNS died due to progressive disease. In another study, Aviles et al. [25] evaluated the impact of a rituximab's (monoclonal antibody against CD20) addition and dose density chemotherapy regimen on the clinical outcome as measured by EFS and OS. In this single arm prospective study, patients with early stage DLBCL-PBL were allocated to receive 6 cycles of chemotherapy CEOP-R (cyclophosphamide $1500 \mathrm{mg} / \mathrm{m}^{2}$ i.v. on day 1 , epirubicin $120 \mathrm{mg} / \mathrm{m}^{2}$ i.v. on day 1 , vincristine $1.2 \mathrm{mg} / \mathrm{m}^{2}$ i.v. on day 1 , prednisone $100 \mathrm{mg} / \mathrm{m}^{2}$ p.o. daily, on day $1-5$, rituximab $375 \mathrm{mg} / \mathrm{m}^{2}$ i.v. on day 1) administered every 14 days. Granulocyte colonystimulating factor (G-CSF) $5 \mu \mathrm{g} / \mathrm{kg} /$ day was started on the second day. There were no differences in either the CR $(87 \%)$ or EFS (63\%) rates, compared to the patients described in their previous study.

Gholam et al. [26] analyzed 34 cases of PBL over a 25-year period. 20 patients were treated with $\mathrm{CHOP}$ or a CHOP-like chemotherapy regimen. All these $20 \mathrm{PBL}$ cases were of B-cell origin (including one case of Burkitt's lymphoma and 2 cases of lowgrade histological type). Sixteen of the 20 patients achieved CR and 2 achieved a partial remission (PR) (> 75\% tumour regression). The remaining two patients had progressive disease during therapy. Within a median follow-up period of 80 months, 6 patients relapsed. Median time from diagnosis to relapse was 23 months (range: 3 to 41 months). Two of the relapses involved the CNS. The neoplasm of two patients who achieved a PR after chemotherapy spread to the CNS. All 4 patients with CNS involvement died. Three of the patients in this study (15\%) developed a contralateral breast relapse. At the time of writing, 12 of the initial 20 patients were alive, including 11 with a persistent CR, 6 patients had died of their lymphomas and 2 of unrelated diseases. In conclusion, they observed a high incidence of CNS relapse in this group of localized extranodal lymphoma. This strongly suggests that CNS prophylaxis should be associated with systemic chemotherapy in localized PBL.

Jeanneret-Sozzi et al. [1] analyzed the clinical profile, treatment, outcome and prognostic factors of 84 patients with PBL, hospitalized in the institutions of the Rare Cancer Network between 1970 and 2000. 21 of the patients underwent a mastectomy, 39 conservative surgery and 23 biopsy. The majority of patients received postoperative treatment: 37 radiotherapy combined with chemotherapy, 22 chemotherapy only, 14 radiotherapy only, whereas 11 patients had no treatment after surgery. The median RT dose was $40 \mathrm{~Gy}$ (range 12-55 Gy). Fifty nine of the 84 patients (70\%) were treated with chemotherapy (the majority with CHOP or CHOP-like regimens). Following this treatment, $12 \%$ of patients 
experienced a local relapse, while a systemic relapse was observed in $55 \%$ of the patients. CNS was the site of relapse in $14 \%$ of the patients. Out of the 12 patients with CNS relapse, $85 \%$ had a high grade lymphoma. The median time to systemic relapse was 22 months (range: 4 to 140). The 5-year OS, lymphoma-specific survival, disease-free survival and local control rates were $53 \%, 59 \%, 41 \%$, and $87 \%$, respectively. The authors conclude that the outcome of PBL is only fair. Local control is excellent with RT or combined modality treatment, but systemic relapses, including those in the CNS, occur frequently.

Jennings et al. [17] published a meta-analysis of 465 PBL cases from 3 decades (1972-2005). Followup ranged from one to 288 months (mean 48 months). The age range was 17 to 95 years (mean 54 years). DLBCL was the most common histological diagnosis (53\%). The patients were included and classified according to the Wiseman and Liao definition [19]. This study found that mastectomy offered no benefit, either in the treatment of PBL or in protection from recurrence. Treatment that included radiation therapy in stage I patients (node negative) showed benefit in both survival $(p=0.002)$ and recurrence rates. Treatment that included chemotherapy in stage II patients (node positive) showed benefits in both survival and recurrence rates. Data regarding combined radiation and chemotherapy were equivocal, due to small sample sizes but, together, they probably had the same survival and recurrence advantages as single treatment options in appropriate node status groups. The authors emphasized the importance of node status for patients with PBL.

In table I we present review of the literature.

\section{Case 1}

A 70-year-old woman underwent cholecystectomy in 2002 and a partial gastrectomy due to peptic ulcer in 2003. Physical examination in February 2005 revealed a tumour in the upperexternal quadrant of her left breast. Detailed physical examination and ultrasonography (USG) revealed axillary lymphadenopathy. Surgery was performed and the tumour and the axillary lymph nodes were removed. Histopathological and immunohistochemical findings in the tumour mass and lymph nodes confirmed the diagnosis of a diffuse large B-cell type primary breast lymphoma: CD20+, CD3-, cytokeratin negative. Paraffin-section studies were positive for $\mathrm{Ki} 67$ in $80 \%$ of neoplasmatic cells. There were no abnormalities in computerized tomography (CT) of the thorax and abdomen. The patient was treated with 6 cycles of CHOP (cyclophosphamide $750 \mathrm{mg} / \mathrm{m}^{2}$ i.v. on day 1 , doxorubicin $50 \mathrm{mg} / \mathrm{m}^{2}$ i.v. on day 1 , vincristine $2 \mathrm{mg}$ i.v. on day 1, prednisone $100 \mathrm{mg}$ p.o. daily, day 1-5) from March to July 2005. Examination in September 2005 indicated complete remission (CR). However in May 2007, she started to complain of weakness, fever, and sweating. On physical examination a palpable lymph node of $2 \mathrm{~cm}$ in diameter was detected in the right axillary tail, and others in the thorax near the aorta, in the upper part of the abdomen and in the liver niche. Additionally, some fluid in the left pleural cavity and pathological changes in the spleen were found. The bone marrow histopathology showed that (thinly distributed) lymphocytes accounted for $50 \%$ of cells. CHOP polychemotherapy combined with rituximab (375 mg/m²) was started. Due to cardiological complications, a decision was taken to withdraw the anthracyclines from the second and third cycles of chemotherapy. After three cycles, no peripheral lymphadenopathy, no pleural fluid, and no changes in the spleen were found. Additionally, a reduction in the size of lymph nodes in the thorax was observed. Due to anaemia and thrombocytopenia, erythrocyte and platelet transfusions were given. After a total of six cycles of chemotherapy the physical examination did not reveal any abnormalities. The CT of the thorax and abdomen was normal. There were no signs in the bone marrow smear and trephine biopsy of infiltration by lymphoma. A second CR was confirmed and 15 months later still persists.

\section{Case 2}

A 55-year-old patient had undergone thyroidectomy and radioiodine therapy due to goiter in 1987. In May 2006, she complained of pain in the left breast which had been present for a few years, but no irregularity was detected during palpation. However, in November 2006 palpation did reveal a mass in the left breast. The patient underwent tylectomy. Macroscopically there were two lymph nodes of $3 \times 2.3 \mathrm{~cm}$ and $3 \times 1.5 \mathrm{~cm}$ together with some fat tissue. Histological and immunohistochemical studies revealed the following: a follicular lymphoma (grade $2 / 3$, predominantly follicular) with bcl2+, CD20+, CD3-, CD43-, CD23-, CD10+, CD5-. There were no abnormalities in the computerized tomography of the thorax and abdomen. There was, however, a palpable lymph node of $0.6 \mathrm{~cm}$ in diameter in the right axillary tail. Bone marrow morphological analysis and flow cytometry analysis did not reveal lymphoma infiltration. Moreover, a trephine biopsy did not reveal any neoplastic cells in the bone marrow. The patient was therefore diagnosed with stage I A lymphoma according to the Ann Arbor classification and CHOP chemotherapy was introduced. Due to the limited stage of the disease, only three cycles of the chemotherapy were given. There were no complications during the treatment. Mammo- 


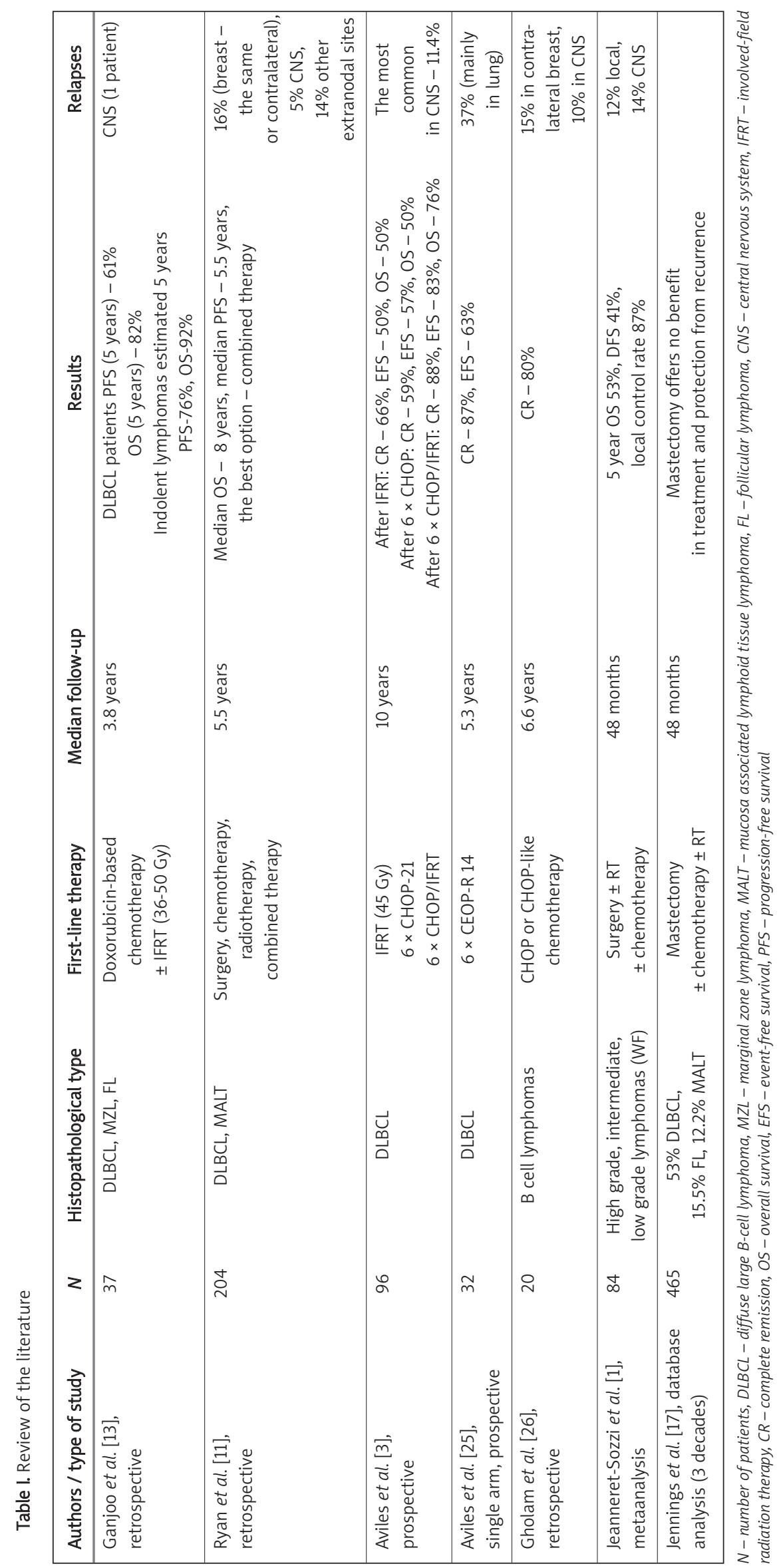


graphy and CT of thorax and abdomen confirmed CR. During an 18 month follow-up period no relapse was observed.

\section{Case 3}

A 58-year-old woman was admitted to our hospital in May 2007 with a clinical diagnosis of lymphoma relapse in her CNS. In 2002, a mass in the external-superior quadrant of the left breast had been detected. Core needle biopsy confirmed the presence of diffuse large B cell lymphoma according to WHO. Paraffin-section studies were positive for CD20, Bcl2, and MIB 1, but negative for CD3. Bone marrow examination and $\mathrm{CT}$ of the thorax and abdomen did not reveal any abnormalities. She received 6 cycles of CHOP chemotherapy. In restaging CR was confirmed, but in May 2007 she complained of headache and confusion. In magnetic resonance (MR) two tumours were revealed in the CNS. One of them, located in the left occipital lobe, was $17 \times 20$ $\times 15 \mathrm{~mm}$ in diameter. The second one $(37 \times 36$ $\times 37 \mathrm{~mm}$ in diameter) was revealed in the left temporal lobe. Radiotherapy of the CNS (40 Gy) was performed. Staging procedures, consisting of a bone marrow examination, $\mathrm{CT}$ of the thorax and abdomen were performed and did not reveal any other sites of relapse. MR of her head showed reduction in the tumour's mass by $75 \%$. We instituted multi-drug chemotherapy with HD MTX and high-dose of arabinoside cytosine (HD AraC) and, after 3 cycles MR revealed a further improvement: occipital tumour had disappeared, while the tumour in the temporal lobe had shrunk to $12 \times 10 \times 8 \mathrm{~mm}$ in diameter. The patient was qualified for autologous stem cell transplantation (ASCT) but unfortunately, mobilization of hematopoietic stem cells failed twice. No symptoms of disease relapse were observed after a follow-up period of 8 months.

In table II we present summary of three cases.

\section{Discussion}

The behaviour of primary lymphoma of the breast is thought to be similar to that of lymphomas of the same histological types and stages arising at other sites. The patients with PBL probably also have a stage-for-stage clinical outcome. The method chosen for treating PBL using radiation therapy and/or chemotherapy also varies in the literature. Currently, the use of combined therapy is considered to be more useful treatment of patients with PBL even in the early stages of their illness $[15,21,27,28]$. Mastectomy is not recommended because it offers no benefit as regards survival or recurrence risk. The most common chemotherapy agents used in PBL have been those in the CHOP regimen. Strategies to minimize the cardiac toxicity risks associated with doxorubicin-containing combination regimens, by using adjuvant radiation therapy, have been reported for non-Hodgkin's lymphoma of sites other than breast and may be important [29].

Rituximab has been registered for treatment of DLBCL and FL. To our knowledge, there are no valuable data that confirm improvement after the addition of rituximab in patients with DLBCL- and FL-PBL. The efficacy of rituximab is well documented in other CD20+ B cell lymphomas. Therefore, prospective trials should be pursued to check the impact of using rituximab on clinical outcome and patterns of relapse. However, it will be difficult due to low incidence of PBL in the general population.

Most authors recommend that aggressive forms of PBL should have CNS prophylaxis even in the early stages. In our opinion, in some forms of $\mathrm{PBL}$ (i.e. with CNS involvement) combination chemotherapy including HD MTX and/or HD Ara-C may improve the outcome and significantly reduce the future risk of CNS relapse. The latest suggestion may be supported by follow-up of our patient with CNS relapse of PBL.

Table II. Summary of presenting cases

\begin{tabular}{|c|c|c|c|}
\hline Sex/age & Histopathological diagnosis & Treatment & Results \\
\hline $\mathrm{F} / 70$ & $\begin{array}{c}\text { DLBCL } \\
(\text { CD20+, CD3-, cytokeratin negative, Ki67 80\%) }\end{array}$ & $\begin{array}{l}6 \times \text { CHOP } \\
6 \times \text { CHOP-R }\end{array}$ & $\begin{array}{l}\mathrm{CR} \text {, relapse } \\
\mathrm{CR}\end{array}$ \\
\hline$F / 55$ & $\begin{array}{c}\mathrm{FL} \text { (grade2/3) } \\
(\mathrm{bcl} 2+, \mathrm{CD} 20+, \mathrm{CD} 3-, \mathrm{CD} 43-, \mathrm{CD} 23-, \mathrm{CD} 10+, \mathrm{CD} 5-)\end{array}$ & $3 \times \mathrm{CHOP}$ & CR \\
\hline$F / 58$ & $\begin{array}{c}\mathrm{DLBCL} \\
(\mathrm{CD} 20+, \mathrm{Bcl} 2+, \mathrm{MIB} 1+, \mathrm{CD} 3-)\end{array}$ & $\begin{array}{c}6 \times \text { CHOP-CR } \\
\text { CNS relapse - } \\
\text { radiotherapy ( } 40 \text { Gy) } \\
\text { and chemotherapy } \\
(3 \times \text { HD MTX/HD Ara C) } \\
\text { HSC mobilization }\end{array}$ & $\begin{array}{c}\text { PR } \\
\text { Ineffective }\end{array}$ \\
\hline
\end{tabular}

$F$-female, $D L B C L$ - diffuse large B-cell lymphoma, $F L$ - follicular lymphoma, CR-complete remission, $P R$ - partial remission, CNS - central nervous system, HD - high doses, HSC - hematopoietic stem cells 


\section{References}

1. Jeanneret-Sozzi W, Taghian A, Epelbaum R, et al. Primary breast lymphoma: patient profile, outcome and prognostic factors. A multicentre Rare Cancer Network study. BMC Cancer 2008; 8: 86.

2. Arber DA, Simpson JF, Weiss LM, Rappaport H. NonHodgkin's lymphoma involving the breast. Am J Surg Pathol 1994; 18: 288-95.

3. Aviles A, Delgado S, Nambo MJ, Neri N, Murillo E, Cleto S. Primary breast lymphoma: results of a controlled clinical trial. Oncology 2005; 69: 256-60.

4. Bobrow LG, Richards MA, Happerfield LC, et al. Breast lymphomas: a clinicopathologic review. Hum Pathol 1993; 24: 274-8.

5. Brogi E, Harris NL. Lymphomas of the breast: pathology and clinical behavior. Semin Oncol 1999; 26: 357-64.

6. Cohen Y, Goldenberg N, Kasis S, Shpilberg D, Oren M. Primary breast lymphoma. Harefuah 1993; 125: 24-6, 63.

7. Domchek SM, Hecht JL, Fleming MD, Pinkus GS, Canellos GP. Lymphomas of the breast: primary and secondary involvement. Cancer 2002; 94: 6-13.

8. Ha CS, Dubey P, Goyal LK, Hess M, Cabanillas F, Cox JD. Localized primary non-Hodgkin lymphoma of the breast. Am J Clin Oncol 1998; 21: 376-80.

9. Kuper-Hommel MJ, Snijder S, Janssen-Heijnen ML, et al. Treatment and survival of 38 female breast lymphomas: a population-based study with clinical and pathological reviews. Ann Hematol 2003; 82: 397-404.

10. Mattia AR, Ferry JA, Harris NL. Breast lymphoma. A B-cell spectrum including the low grade B-cell lymphoma of mucosa associated lymphoid tissue. Am J Surg Pathol 1993; 17: 574-87.

11. Ryan G, Martinelli G, Kuper-Hommel M, et al. Primary diffuse large B-cell lymphoma of the breast: prognostic factors and outcomes of a study by the International Extranodal Lymphoma Study Group. Ann Oncol 2008; 19: 233-41.

12. Topalovski M, Crisan D, Mattson JC. Lymphoma of the breast. A clinicopathologic study of primary and secondary cases. Arch Pathol Lab Med 1999; 123: 1208-18.

13. Ganjoo K, Advani R, Mariappan MR, McMillan A, Horning S. Non-Hodgkin lymphoma of the breast. Cancer 2007; 110: 25-30.

14. Kim SH, Ezekiel MP, Kim RY. Primary lymphoma of the breast: breast mass as an initial symptom. Am J Clin Oncol 1999; 22: 381-3.

15. Dao AH, Adkins RB Jr, Glick AD. Malignant lymphoma of the breast: a review of 13 cases. Am Surg 1992; 58: 792-6.

16. Zack JR, Trevisan SG, Gupta M. Primary breast lymphoma originating in a benign intramammary lymph node. AJR Am J Roentgenol 2001; 177: 177-8.

17. Jennings WC, Baker RS, Murray SS, et al. Primary breast lymphoma: the role of mastectomy and the importance of lymph node status. Ann Surg 2007; 245: 784-9.

18. Yoshida S, Nakamura N, Sasaki Y, et al. Primary breast diffuse large B-cell lymphoma shows a non-germinal center B-cell phenotype. Modern Pathology 2005; 18: 398-405.

19. Gualco G, Bacchi CE. B cell and T-cell lymphomas of the breast:clinical-pathological features of 53 cases. Int Surg Pathol 2008; 16: 407-13.

20. Wong AK, Lopategiu J, Clancy S, Kluber D, Bose S. Anaplstic large cell lymphoma associated with a breast implant capsule: a case report and review of the literature. Am J Surg Pathol 2008; 32: 1265-8
21. Wiseman C, Liao KT. Primary lymphoma of the breast. Cancer 1972; 29: 1705-12.

22. Lyou CY, Yang SK, Choe DH, Lee BH, Kim KH. Mammographic and sonographic findings of primary breast lymphoma. Clin Imaging 2007; 31: 234-8.

23. Lin Y, Guo XM, Shen KW, Wang JL, Jiang GL. Primary breast lymphoma: long-term treatment outcome and prognosis. Leuk Lymphoma 2006; 47: 2102-9.

24. Hugh JC, Jackson FI, Hanson J, Poppema S. Primary breast lymphoma. An immunohistologic study of 20 new cases. Cancer 1990; 66: 2602-11.

25. Aviles A, Castaneda C, Neri N, Cleto S, Nambo MJ. Rituximab and dose dense chemotherapy in primary breast lymphoma. Haematologica 2007; 92: 1147-8.

26. Gholam D, Bibeau F, El Weshi A, Bosq J, Ribrag V. Primary breast lymphoma. Leuk Lymphoma 2003; 44: 1173-8.

27. Kelenyi G. Malignant lymphomas of the breast. Zentralbl Pathol 1991; 137: 264-9.

28. Wong WW, Schild SE, Halyard MY, Schomberg PJ. Primary non-Hodgkin lymphoma of the breast: The Mayo Clinic Experience. J Surg Oncol 2002; 80: 19-25.

29. Miller TP, Dahlberg S, Cassady JR, et al. Chemotherapy alone compared with chemotherapy plus radiotherapy for localized intermediate- and high-grade non-Hodgkin's lymphoma. N Engl J Med 1998; 339: 21-6. 